\title{
Quantitative Experimental and Theoretical Investigations on the Interaction of Shock Waves with Magnetic Fields
}

\author{
H. KLINGENBerG * F. SARdei, and W. ZimmermanN \\ Institut für Plasmaphysik, Garching bei München, Germany
}

(Z. Naturfcrsch. 24 a, 1449-1457 [1969] ; received 24 June 1969)

\begin{abstract}
In continuation of the work on interaction between shock waves and magnetic fields ${ }^{1,2}$ the experiments reported here measured the atomic and electron densities in the interaction region by means of an interferometric and a spectroscopic method.

The transient atomic density was also calculated using a one-dimensional theory based on the work of JoHNSON ${ }^{3}$, but modified to give an improved physical model. The experimental results were compared with the theoretical predictions.
\end{abstract}

The published results ${ }^{1,2}$ of investigations on the interaction of a shock-heated plasma flow with a perpendicular magnetic field using several electrode pairs has shown that the high currents necessary for strong interaction can only be produced in the form of arcs. With arcs it is difficult to satisfy the simple conditions of one-dimensional theory, such as uniform current distribution in the interaction region. In order to achieve at least an approximately uniform current distribution the arc phenomenon was utilized by mounting many electrodes of small surface area alongside and behind one another flush in the wall and short-circuiting the pairs separately, thus producing many arcs alongside and behind one another. With this configuration strong interaction effects were observed.

Streak photographs showed a deceleration of the plasma flow and also luminous fronts running counter to the flow which were reflected from the contact front, at least at low inital pressures. At high magnetic field strengths these luminous fronts left the interaction region in the upstream direction, while at low magnetic field strengths they remained in the interaction region. These fronts were interpreted as shock fronts. Effects of rarefaction waves were also observed.

To obtain further information on these observations quantitative investigations were begun. This paper describes results of interferometric and spectroscopic measurements of the atomic and electron densities in the interaction region. To compare experimental results with theory, a transient onedimensional theory based on the theoretical work of JoHNSON ${ }^{3}$ was developed.

Reprint requests to: Institut für Plasmaphysik, D-8046 Garching bei München.

* Now with Volkswagenwerk AG, Wolfsburg, Germany.

\section{Theory}

Since the results of previous investigations ${ }^{1,2}$ showed that the current density is governed by the complicated boundary conditions in the plasma-toelectrode transition region, the current density, which is an important parameter of the theory, cannot be expressed in such a simple way as in Ref. ${ }^{3}$. The resistance to the discharge current arises mainly in the boundary layer. In this way part of the energy of the main flow region enters the transition region. There is therefore energy output from the main flow, even if the external resistance of the electrodes and the bridge connecting the electrodes is negligible. The resistance of the transition region can be regarded as an additional external resistance. The main plasma flow region forms the internal resistance. The thickness of the transition region is very small compared with the diameter of the main flow and hence the interaction between magnetic field and the flow can still be described satisfactorily by a simple one-dimensional model. However, because there is no theory describing the conditions in the boundary layer, the current density cannot be calculated, but has to be taken from experimental results. This current density is assumed to be uniform and transverse to the flow direction in the interaction region. From experimental results ${ }^{1,2}$ it is known that the induced magnetic field can be neglected relative to the external magnetic field, which is perpendicular to the flow direction and the current. The following theory takes this conditions into account. Furthermore, thermal conductivity and viscosity is assumed to be zero and radiation losses

1 H. Klingenberg, Z. Naturforsch. 23 a, 1929 [1968].

2 H. Klingenberg, Z. Naturforsch. 24 a, 540 [1969].

3 M. R. Johnson, Phys. Fluids 10, 539 [1967]. 
are neglected. Ionization is not taken into account but a constant conductivity is assumed.

The one-dimensional interaction is then described by the three equations of conservation of mass, momentum and energy, by the equation of state, and by Ohm's law without the Hall term.

If one uses the equation of state

$$
p=\varrho R T
$$

and Ohm's law

$$
j=\sigma\left(E+v B_{0}\right)
$$

to eliminate the temperature $T$ and electric field $E$ from the energy equation, one can write the three conservation equations in the following dimensionless form:

$$
\begin{aligned}
& \frac{\partial D}{\partial \tau}+D \frac{\partial V}{\partial X}+V \frac{\partial D}{\partial X}=0 \\
& D \frac{\partial V}{\partial \tau}+V D \frac{\partial V}{\partial X}+Q_{1} \frac{\partial P}{\partial X}+N_{1} J=0 \\
& \frac{\partial P}{\partial \tau}+V \frac{\partial P}{\partial X}-\frac{P}{D} \frac{\partial D}{\partial \tau}-\frac{V P}{D} \frac{\partial D}{\partial X} \\
&+(\gamma-1)\left[P \frac{\partial V}{\partial X}-\frac{N_{1} J^{2}}{Q_{1}}\right]=0
\end{aligned}
$$

with

$$
\begin{gathered}
\tau=t v_{1} / L ; \quad X=x / L ; \\
D=\varrho / \varrho_{1} ; P=p / p_{1} ; V=v / v_{1} ; J=J(X, \tau) ; \\
Q_{1}=p_{1} /\left(\varrho_{1} v_{1}^{2}\right) ; \quad N_{1}=\sigma_{1} B_{0}^{2} L /\left(\varrho_{1} v_{1}\right) ;
\end{gathered}
$$

$\gamma=$ ratio of specific heats, $L=$ length of interaction region, $x=$ distance measured from the beginning of the interaction region, $t=$ time counted from the entrance of the primary shock into the interaction region, $\varrho, p, v$ are density, pressure and flow velocity respectively, $\sigma=$ conductivity. The subscript 1 denotes the values in the region behind the unperturbed primary shock front before this front enters the interaction region, $B_{0}=$ external magnetic field.

$J(X, \tau)$ is the dimensionless current density which can be explained as follows: When the shock front enters the interaction region the current does not rise discontinuously (as assumed by $\mathrm{JOHNSON}^{3}$ ), but obeys essentially an exponential law (starting from zero behind the primary shock front) because of the inductance of the circuit. Behind the primary shock front the current density $J(X, \tau)$ is therefore assumed to obey the law:

$$
\begin{array}{ll}
J(X, \tau)=J_{\max } \cdot\left(1-\exp \left\{-\left[\tau-\tau_{\text {shock }}(X)\right] / K\right\}\right) \\
\text { with } \quad J_{\max }=j_{\max } /\left(\sigma_{1} v_{1} B_{0}\right)
\end{array}
$$

for the region $0 \leqq X \leqq 1 ; \tau \leqq \tau_{\text {shock }}(X)$ in the $X-\tau$ plane, see Fig. 1 .

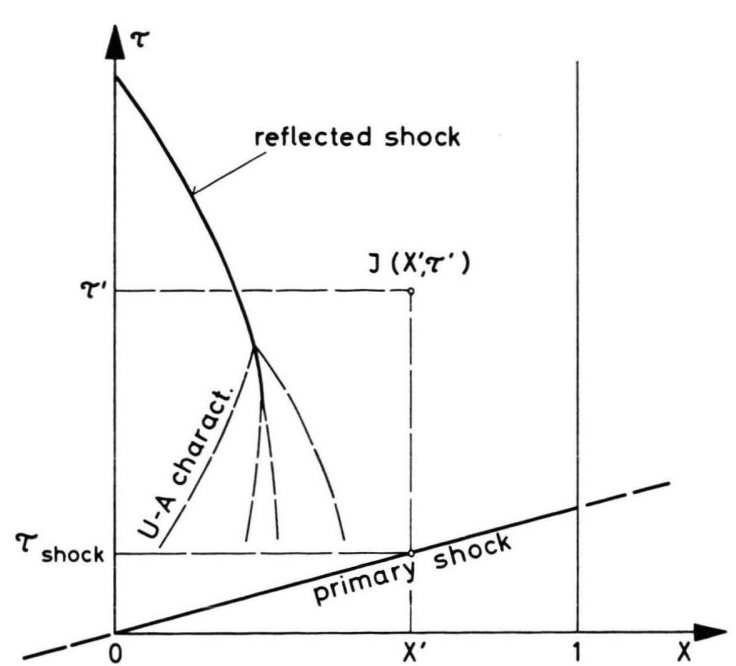

Fig. 1. Primary shock and formation of the reflected shock from the U-A characteristics (schematically). The value of the current density at the point $J\left(X^{\prime}, \tau^{\prime}\right)$ depends on the parameter $\tau$ shock $\left(X^{\prime}\right)$ according to Eq. (7).

$j_{\max }$ and the time constant $K$ are taken from experimental results ${ }^{1,2}, \sigma_{1}, \varrho_{1}, p_{1}$ and $v_{1}$ are taken from calculations ${ }^{4}$. For $X>1$ and $X<0 J$ is assumed to be 0 .

The Eqs. (3) to (5) with the dependent variables $P, D, V$ can now be solved by the characteristic method. The three characteristic directions are

$$
\mathrm{d} X / \mathrm{d} \tau=V \quad \text { and } \quad \mathrm{d} X / \mathrm{d} \tau=V \pm A .
$$

The following differential equations are valid along the respective directions:

$Q \mathrm{~d} P-A^{2} \mathrm{~d} D-(\gamma-1) N_{1} J^{2} \mathrm{~d} \tau=0 \quad$ and

$$
Q \mathrm{~d} P \pm A D \mathrm{~d} V+N_{1} J[(1-\gamma) J \pm A] \mathrm{d} \tau=0
$$

with $A=a / v_{1}$ ( $a=$ local sonic velocity).

This system (10) can be solved by an iterative procedure. Additional boundary conditions have to be satisfied for the primary shock and for the secondary (reflected) shock, the latter possible being formed as a result of interaction. These boundary conditions are described by Rankine-Hugionot relations in the differential form. The results of the calculations are given in Section IV.

\section{Experimental Arrangement}

The apparatus for the interaction experiment has already been described in Ref. ${ }^{1}$. The shock tube is of

\footnotetext{
${ }^{4} \mathrm{H}$. Carls and L. Rehder, personal communication from the Institut für Experimentalphysik der Universität Kiel (Thesis H. CARLS).
} 
the diaphragm type. The driving gas is hydrogen (100 atm), and the test gas is argon (initial presure: 0.5 to 10 Torr). The measuring chamber $(9 \mathrm{~m}$ from the diaphragm region) is made of plastic sections secured between tie plates and has a square internal cross section of $7 \times 7 \mathrm{~cm}^{2}$. A sharp cutter is used to cut out the rectangular flow from the circular flow of the shock tube.

One of the sections is made of epoxy resin reinforced with fibre-glass and has electrodes and high-grade glass windows for interferometric measurements. Twenty-five pairs of electrodes $4.5 \mathrm{~mm}$ in diameter are used. They are each short-circuited separately with a copper bridge. The currents from the plasma are measured with Rogowski coils, cf. ${ }^{1}$. The magnetic field is produced with a crowbarred capacitor bank via Helmholtz coils. The magnetic field strength is $6.8 \mathrm{kG}$.

Two-wavelength streak interferometry is used for measuring the electron and atomic densities simultaneously as functions of time, see ${ }^{5}$. Single-picture interferograms give the spatial information. The experimental arrangement for the interferometric measurements is shown in Fig. 2 a. A pressurized spark is discharged with 2 msec duration and with $3 \mathrm{kA}$ through a capillary containing argon. The current is generated by a pulse-shaping network. The spark serves as a point source of light for a Mach-Zehnder interferometer (C a rl Z e is s, Oberkochen). The interference beam leaving the interferometer is divided by a beam splitter and the parts are sent via deflecting mirrors through separate filters (wavelength $4840 \AA$ and $6610 \AA$ respectively; half-width $60 \AA$ ) to a slit. The interference pat- terns and the measuring chamber are imaged on this slit. In order to obtain images of equal size the optical path lengths of each of the beams are made equal by choosing an appropriate thickness of the beam splitter. Beneath the slit there is a hole used for imaging two short-duration sparks triggered at different times, the difference being measured with a photomultiplier and a counter. The slit and the hole are focused on the film of a drum camera (Beckman-Whitley), thus allowing streak interferograms at two wavelengths and providing two time marks for giving the velocity of the rotating film. - The interference beam can also be sent to an image converter camera to obtain single pictures (dashed part of Fig. 2 a).

To confirm the results of interferometric measurements a well-known independent method is used to measure the electron density. The absolute value of the continuum radiation of the plasma is measured spectroscopically at different wavelengths. The experimental arrangement is shown in Fig. 2 b. Two different points of the axis of the measuring chamber are focused simultaneously on two light pipes leading to the slit of a spectroscope (Steinheil, Model $6 \mathrm{H}$ ). For each point the intensity at two different wavelengths is measured as a function of time by means of photomultipliers and oscilloscopes. For calibration the light from the anode of a carbon arc is focused on the axis of the measuring chamber and hence on the light pipes cf. ${ }^{6}$, allowance being made for losses due to reflection from the windows and objective surfaces. The optical system has a spatial resolution of about $2 \mathrm{~mm}$. The measurements are restricted to the four points in the centre between the five rows of electrode bridges.

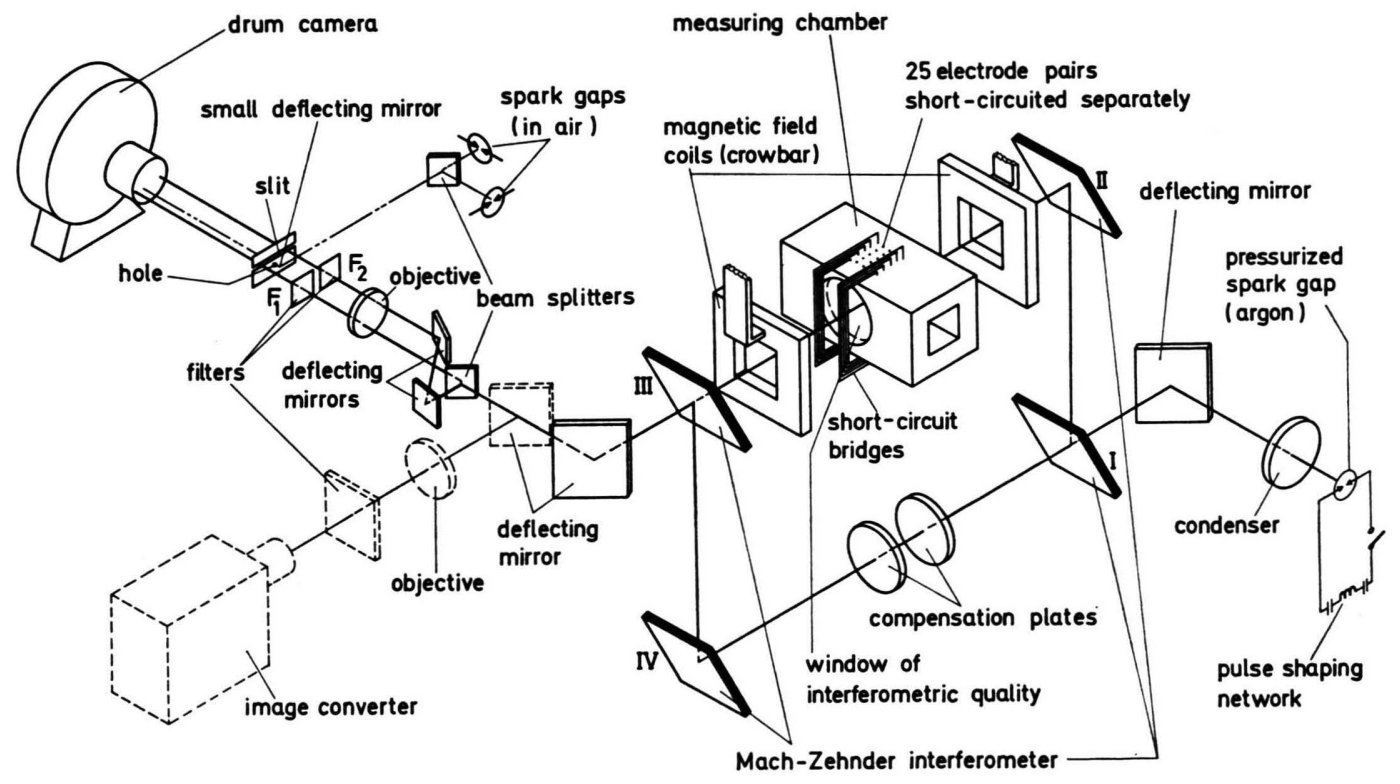

Fig. 2 a. Experimental arrangement for the interferometric measurements. 


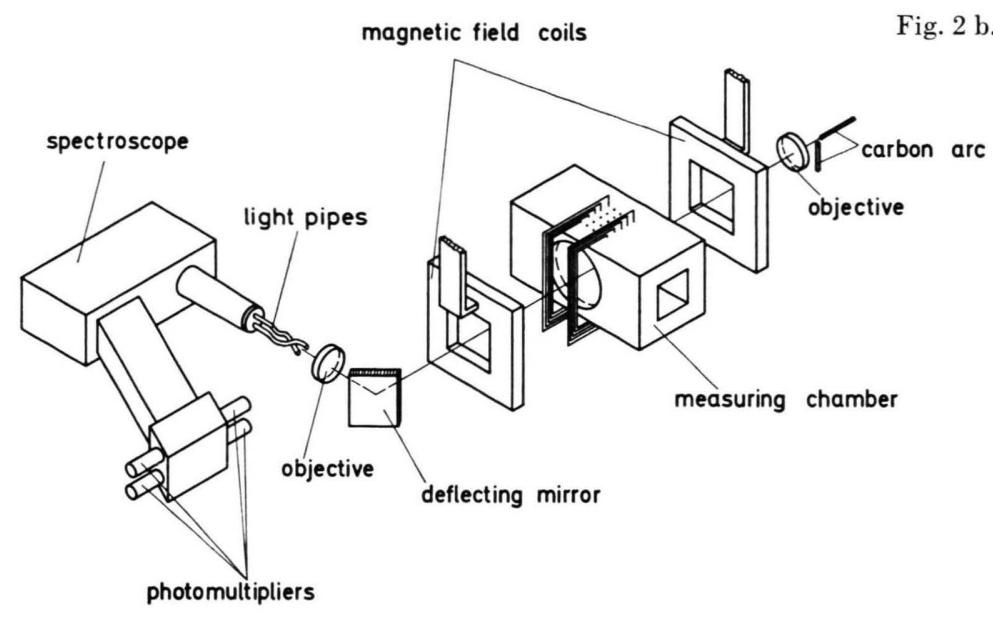

\section{Evaluation of results}

\subsection{Electron density}

For evaluation of the electron density from the measured continuum radiation a formula given by HorN et al. ${ }^{7}$ is used. This is based on a formula by UNsöLD ${ }^{8}$ for hydrogen like atoms:

with

$$
v<v_{\mathrm{g}}
$$

where $\varepsilon_{v}=$ emission coefficient (continuum losses per unit volume and per unit frequency), $C=$ constant given by UNSÖLD ${ }^{8}, Z=$ effective nuclear charge, $N_{\mathrm{e}}=$ electron density, $T=$ temperature, $v_{\mathrm{g}}=$ "cutoff'-frequency.

The $Z$ of argon is taken from calculations made by SCHLÜTER ${ }^{9}$, using the graph in Ref. ${ }^{7} . T$ is assumed to be $15000{ }^{\circ} \mathrm{K}$. (The calculated temperature behind the shock front without current but with allowance for ionization effects is $10000{ }^{\circ} \mathrm{K}$. The error entailed in choosing a rough approximation of the temperature is small compared with other errors.)

The two wavelengths at which the measurements are made simultaneously are selected from timeintegrated spectrograms to avoid radiation of spectral lines and to avoid coincidence with discontinuities of the $Z^{2}$-curve, cf. ${ }^{7}$.

The wavelengths chosen are

$$
\lambda_{1}=5390 \AA, \quad \lambda_{2}=4655 \AA .
$$

8 A. Unsöld, Ann. Phys. Leipzig 33, 607 [1938].

9 D. SCHLÜTER, Z. Astrophys. 61, 67 [1965].
* Fig. 3 a - c, 4 on Table 1452 a, b.

7 K. P. Horn, H. Wong, and D. Bershader, J. Plasma Phys. 1, 157 [1967]. 
H. Klingenberg, F. Sardei, and W. Zimmermann, Quantitative Experimental and Theoretical Investigations on the Interaction of Shock Waves with Magnetic Fields (S. 1449).

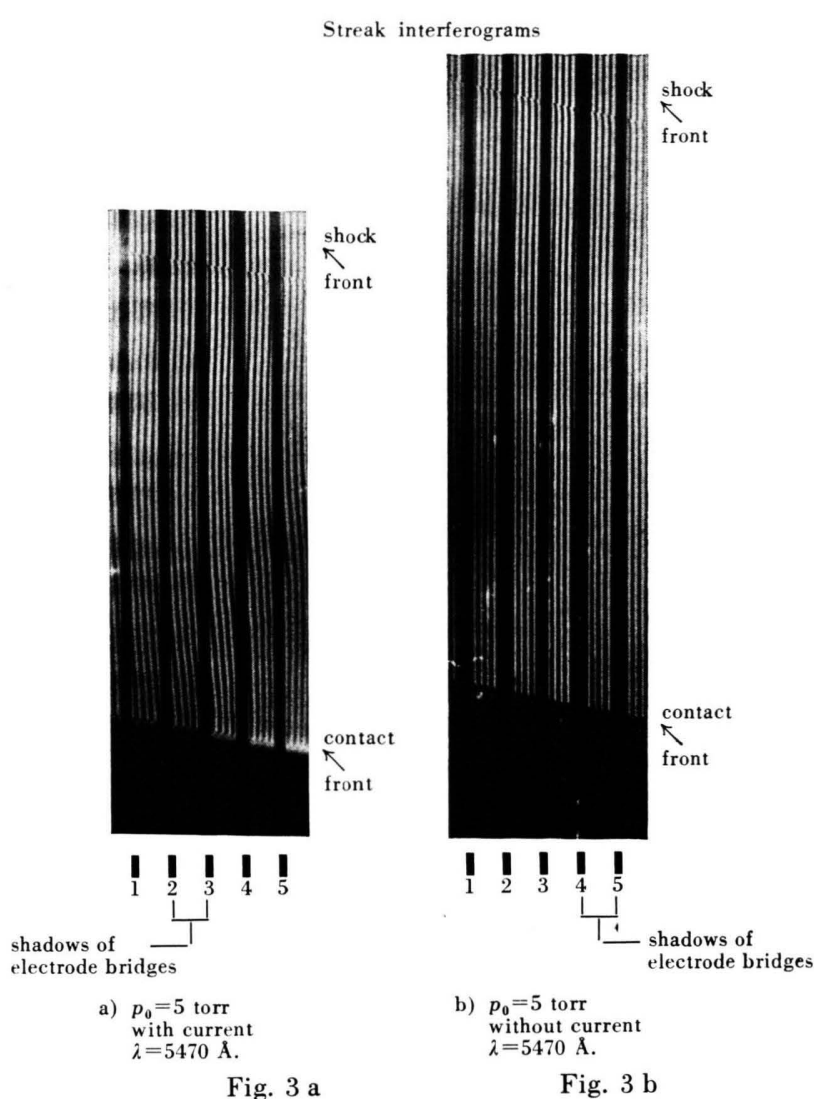

c) $\lambda=5470 \AA ; p_{0}=5$ torr taken with image converter camera

exposure time: $0.1 \mu \mathrm{sec}$ time after arrival of shock front at 1st. row of electrodes: 220 usec

Fig. $3 \mathrm{~b}$

Single-picture interferogram

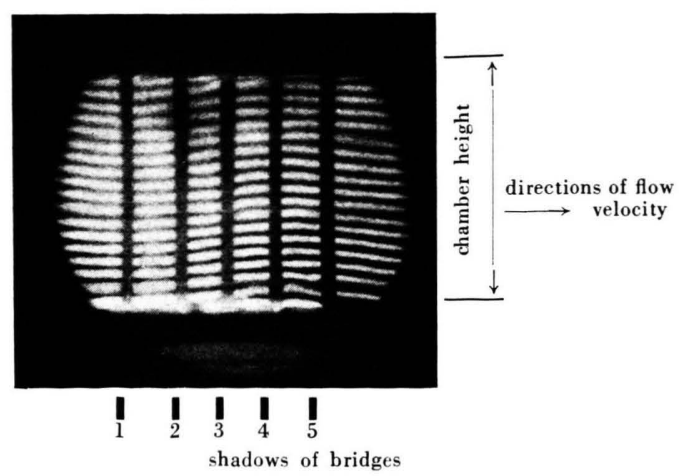

Fig. $3 \mathrm{c}$

Fig. 3. Interferograms of the argon plasma flow in the interaction region. 

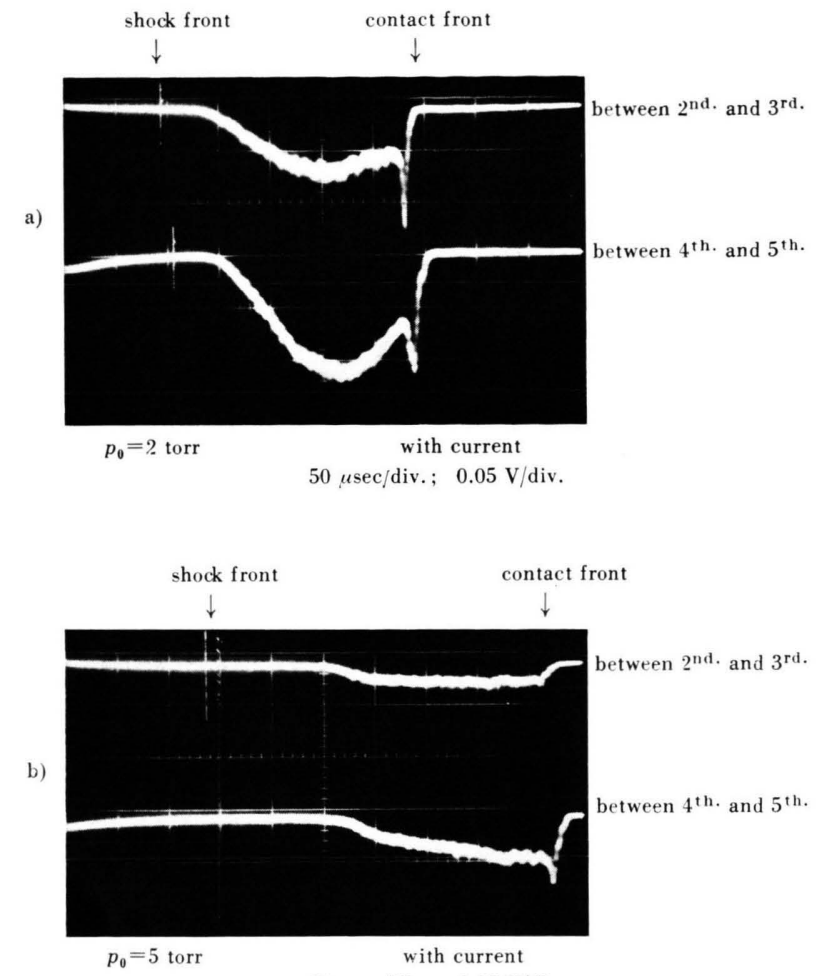

$50 \mu \mathrm{sec} /$ div.; $0.05 \mathrm{~V} /$ div.

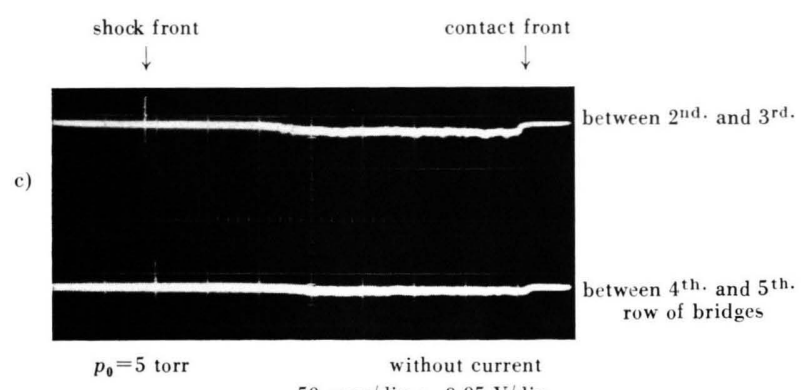
$50 \mu \mathrm{sec} /$ div. ; $0.05 \mathrm{~V} /$ div.

Fig. 4. Multiplier oscillograms of continuum radiation. 
The measured values at both wavelengths from several shots are used to form a mean value. (At $\lambda=4655 \AA$ the measured $N_{\mathrm{e}}$-values are about $10 \%$ lower than those measured at $\lambda=5390 \AA$.)

\subsection{Atomic density}

The formulas given by ALPHER and WHITE can be used ${ }^{5}$ for evaluating the particle densities from measured fringe shifts $s$ :

$$
s=L \cdot \Delta n / \lambda
$$

where $L=$ thickness of plasma slab, $\Delta n=$ change of refractivity, $\lambda=$ wavelength.

The fringe shifts caused by changes in the density of electrons and argon atoms are for a plasma slab thickness of $7 \mathrm{~mm}$ (and the wavelengths measured in $\mathrm{cm}$ )

$$
\begin{gathered}
s\left(N_{\mathrm{e}}\right)=-3.12 \times 10^{-13} \lambda \Delta N_{\mathrm{e}}, \\
s\left(N_{\mathrm{A}}\right)=+7.26 \times 10^{-23}(1 / \lambda) \Delta N_{\mathrm{A}}
\end{gathered}
$$

respectively. $N_{\mathrm{e}}$ and $N_{\mathrm{A}}$ are the number densities of the electrons and the heavy particles (neutral and ionized argon atoms) respectively. Since the refractivity of the ionized atoms is nearly the same as that of neutral atoms, this assumption is acceptable, cf. ${ }^{5}$.

The fringe shift caused by the electrons varies linearly with the wavelength, while the fringe shift caused by the atoms varies linearly with the reciprocal of the wavelength. To determine the electron and atomic densities simultaneously, measurements have to be made at two different wavelengths. In these experiments the wavelengths

$$
\lambda_{1}=4840 \AA \quad \text { and } \quad \lambda_{2}=6610 \AA
$$

are used. So the changes in the electron and atomic densities are given by

$$
\begin{aligned}
\Delta N_{\mathrm{e}}=1.68 \times 10^{17} \cdot\left(0.661 \cdot s_{\lambda=}\right. & 6610 \mathrm{~A}-0.484 \\
& \left.\cdot s_{\lambda=4840 \mathrm{~A}}\right)\left[\mathrm{cm}^{-3}\right], \\
\Delta N_{\mathrm{A}}=2.18 \times 10^{18} \cdot\left(0.661 \cdot \mathrm{s}_{\lambda=}\right. & 4840 \mathrm{~A}-0.484 \quad(14 \\
& \left.\cdot s_{\lambda=6610 \mathrm{~A}}\right)\left[\mathrm{cm}^{-3}\right] .
\end{aligned}
$$

The errors amount to

$$
\begin{aligned}
& \Delta\left(\Delta N_{\mathrm{e}}\right) \approx \pm 1.9 \times 10^{17} \Delta s\left[\mathrm{~cm}^{-3}\right], \\
& \Delta\left(\Delta N_{\mathrm{A}}\right) \approx \pm 2.5 \times 10^{18} \Delta s\left[\mathrm{~cm}^{-3}\right]
\end{aligned}
$$

where $\Delta s$ is the error in determining the fringe shift. In the presented case this error is approximately 0.1 resulting in

$$
\begin{aligned}
& \Delta\left(\Delta N_{\mathrm{e}}\right) \approx \pm 1.9 \times 10^{16}\left[\mathrm{~cm}^{-3}\right], \\
& \Delta\left(\Delta N_{\mathrm{A}}\right) \approx \pm 2.5 \times 10^{17}\left[\mathrm{~cm}^{-3}\right] .
\end{aligned}
$$

It is found from the interferometric measurements that the electron density is of the order of the error value. Therefore the electron density was measured by the spectroscopic method too and from this the fringe shift caused by the electrons was calculated.

The measured total fringe shift is

$$
s=s\left(N_{\mathrm{A}}\right)+s\left(N_{\mathrm{e}}\right) .
$$

After measuring the total fringe shift $s$ at a wavelength $\lambda_{1}=4860 \AA$ and the change in the electron density $\Delta N_{\mathrm{e}}$ by spectroscopic means the change in the density of the atoms can be calculated with help of the following equation which results from (13) and (17):

$$
\Delta N_{\mathrm{A}}=6.7 \times 10^{17} s_{\lambda_{1}}+10.0 \Delta N_{\mathrm{e}}\left[\mathrm{cm}^{-3}\right] .
$$

The total density of the heavy particles is then

$N=N_{0}+\Delta N_{\mathrm{A}}=N_{0}+6.7 \times 10^{17} s_{\lambda_{1}}+10.0 N_{\mathrm{e}}\left[\mathrm{cm}^{-3}\right]$

( $N_{0}=$ atomic density ahead of primary shock front, $\Delta N_{\mathrm{e}}=N_{\mathrm{e}}$ as there are no electrons ahead of the primary shock).

The error in evaluating the fringe shift is $\Delta s \approx 0.1$ and in measuring the electron density is $\Delta\left(N_{\mathrm{e}}\right) \approx \pm 0.4 \times 10^{16}$, resulting in

$$
\Delta\left(\Delta N_{\mathrm{A}}\right) \approx \pm 10^{17}\left[\mathrm{~cm}^{-3}\right] .
$$

At the other wavelength $\lambda_{2}=6610 \AA$ there is a similar formula, but at this wavelength the error is greater by a factor of about 1.5. Therefore, the values obtained at this wavelength were only used for checking the other ones.

The error of $\pm 10^{17}\left[\mathrm{~cm}^{-3}\right]$ (20) found by using the spectroscopic method (18) is a factor of about 2 lower than that [see Eq. (16)] in the evaluation using the two-wavelength method i. e. Eq. (14).

The mass density is

$$
\varrho=N \cdot m_{\mathrm{A}}=N \cdot 6.63 \times 10^{-23}\left[\mathrm{gr} / \mathrm{cm}^{-3}\right] .
$$

\section{Results of spectroscopic measurements}

To test the spectroscopic method the first measurements of the electron density were made on the shock-heated plasma without magnetic field, i. e. without currents flowing. The electron density of the shock-heated plasma can in this case be calculated for the equilibrium state, cf., for example ${ }^{4,10,11}$.

10 H. Klingenberg and H. Muntenbruch, Report IPP 3/45, Nov. 1966.

11 H. NeTt, Report IPP 3/43, October 1966. 
Figure 5 shows the calculated and measured values for equilibrium conditions. The error spreads of the calculated values are determined by the error in the measurements of the shock Mach number.

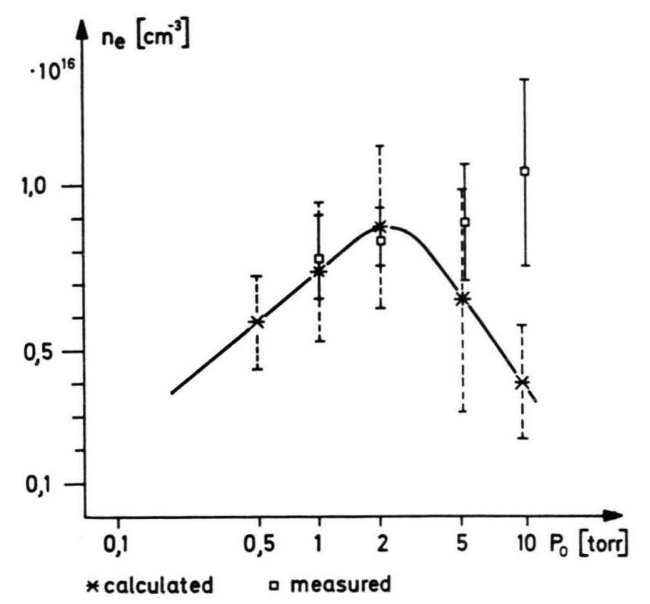

Fig. 5. Calculated and measured values of electron density of shock-heated plasma vs. initial pressure (equilibrium, no magnetic field).
At low initial pressures the values agree fairly well. With increasing initial pressure starting from $p_{0}=5$ Torr, the measured values increase, while the calculated values decrease. At $p_{0}=5$ Torr error spreads will overlap. These results show that the method is reliable at least up to this pressure. The discrepancy at higher initial pressures is presumably caused by the impurities producing the secondary fronts in the streak pictures, cf. ${ }^{1,2}$. (Very small particles are swept up and carried along by the flow.)

With current flowing, the electron density is higher compared with the former case. Figure 6 shows, as an example, the measured electron density in three dimensional representation vs. time $t$ and distance $x$ for $p_{0}=5$ Torr. The coordinates correspond to those of Fig. 8 . The measuring points are situated in the middle between the rows of the electrodes.

The electron density increases in the downstream direction, but more distinctly towards the end of the interaction region, thus demonstrating the influence

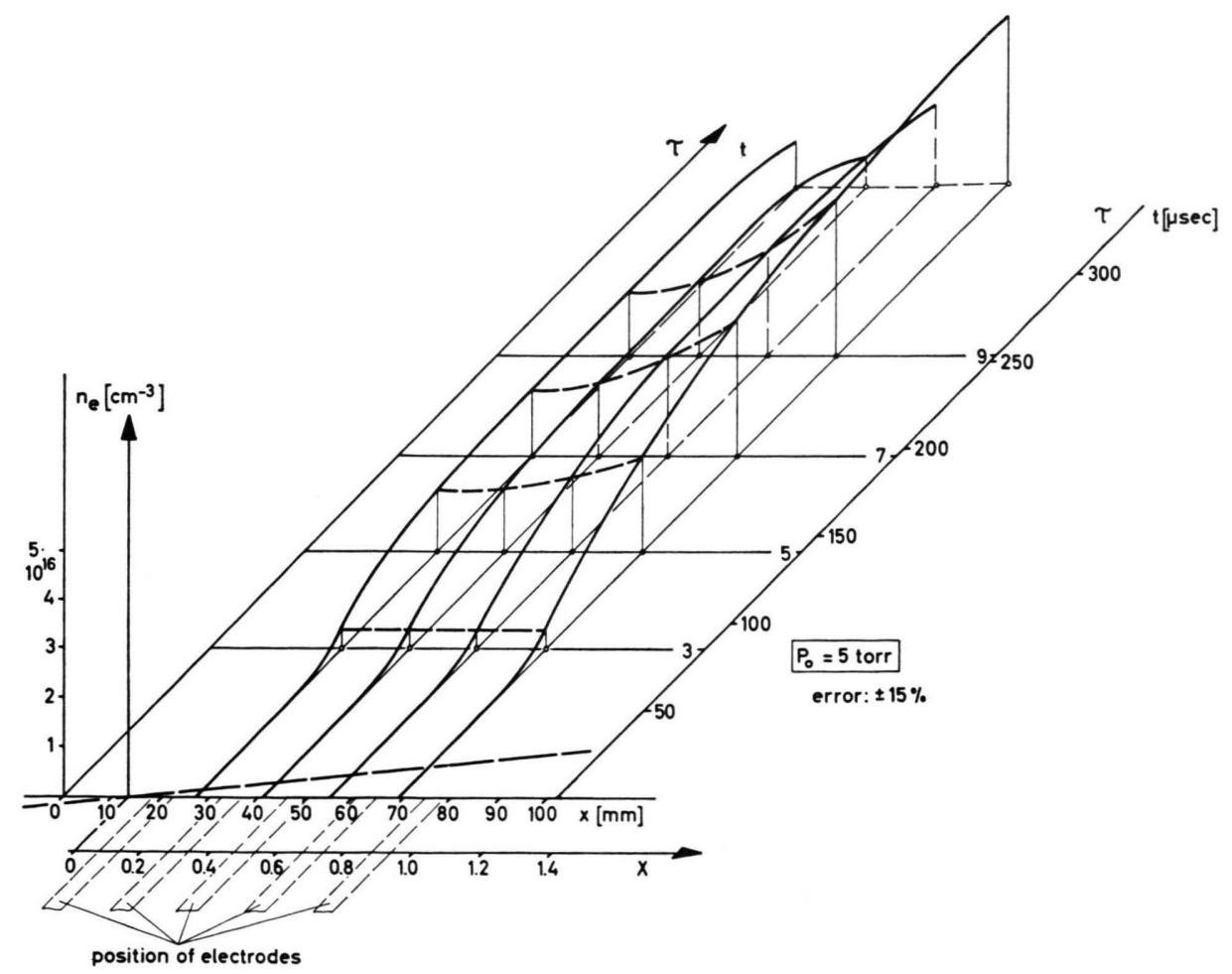

Fig. 6. Measured electron density vs. time and distance; $p_{0}=5$ Torr. The origin of the time coordinate corresponds to the arrival of the shock front at the upstream boundary of the interaction region. The interaction region is between 13 and $83 \mathrm{~mm}$. 
of the currents. The increase with time is especially pronounced at the last measuring point betwen the fourth and the fifth rows of electrodes. The maximum value is about three times the equilibrium value without current.

\section{Results of interferometric measurements}

To check the interferometric method, measurements of the atomic density were made immediately behind the primary shock front. The ionization in this region is low, because of the relaxation length in argon and the atomic density can be calculated by solving the Rankine-Hugoniot relations without taking ionization effects into account, cf. ${ }^{10,11}$. Figure 7 shows the calculated and measured atomic densities immediately behind the shock front. The values agree within the estimated errors.

Figure 8 a shows as an example the measured atomic density with current flowing as a function of the time $t$ and the distance $x$ for $p_{0}=5$ Torr. The curves represent mean values taken from three shots. (Measurements at initial pressures below 2 Torr

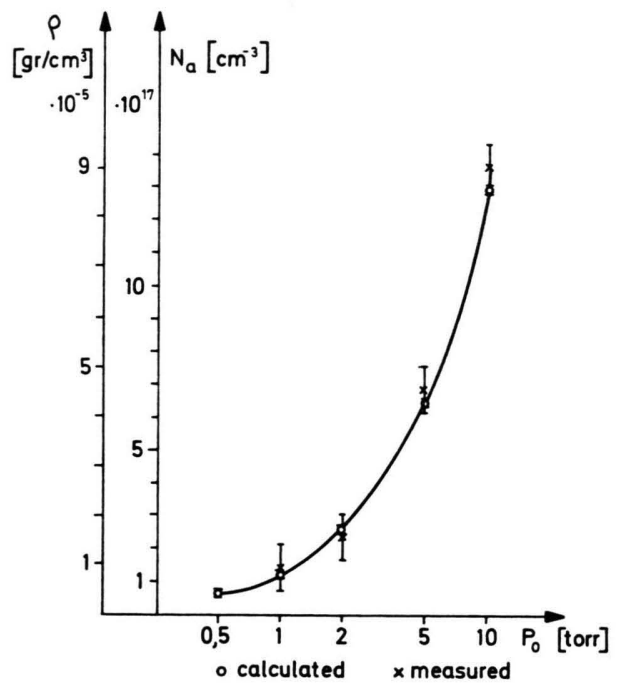

Fig. 7. Atomic density immediately behind the shock front vs. initial pressure of the argon test gas.

gave no useful result because the density was too low and because the error was too large.)

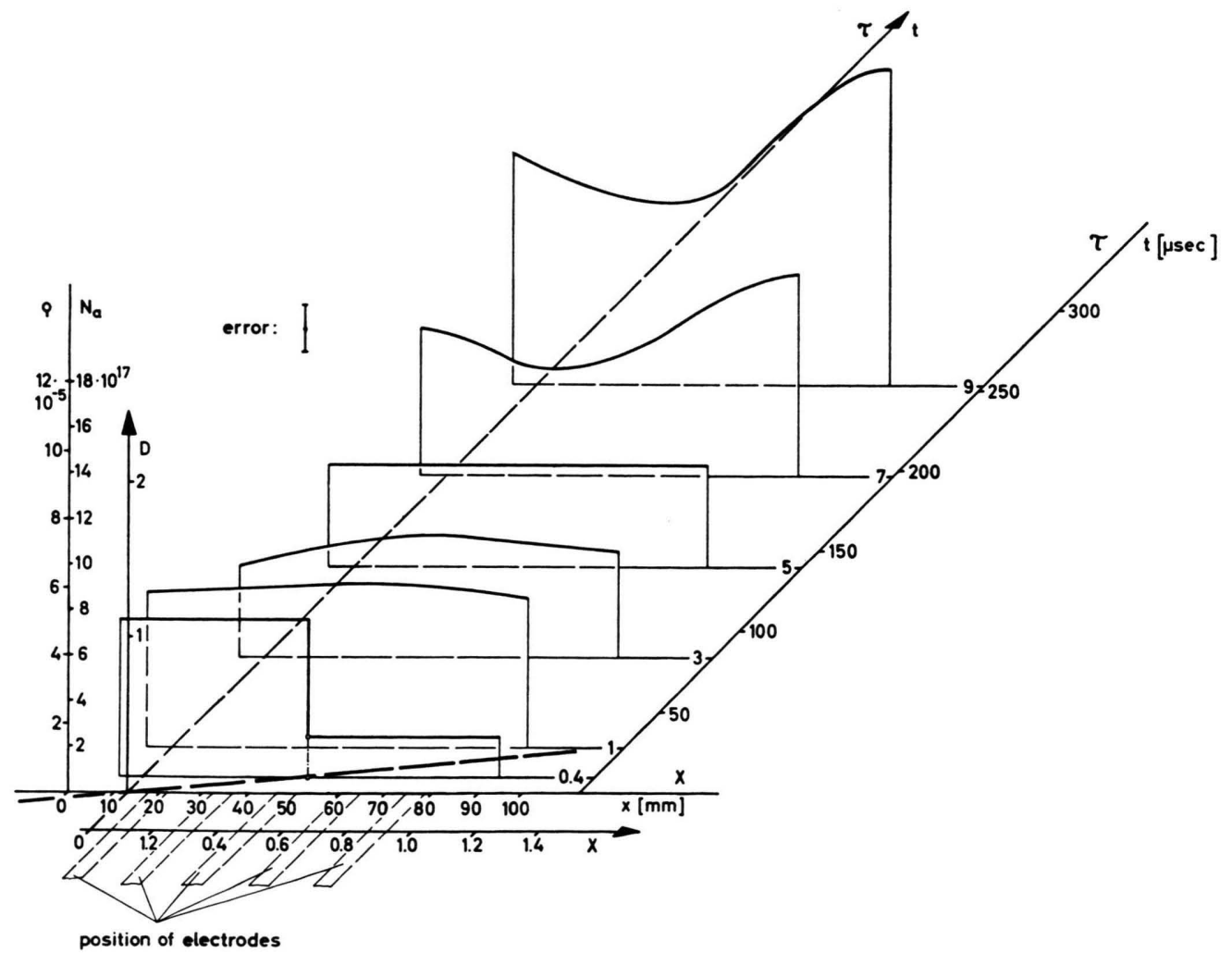

Fig. 8 a. Measured atomic density vs. time and distance; $p_{0}=5$ Torr. The origin of the time coordinate corresponds to the arrival of the shock front at the upstream boundary of the interaction region. Interaction region as in Fig. 6. 


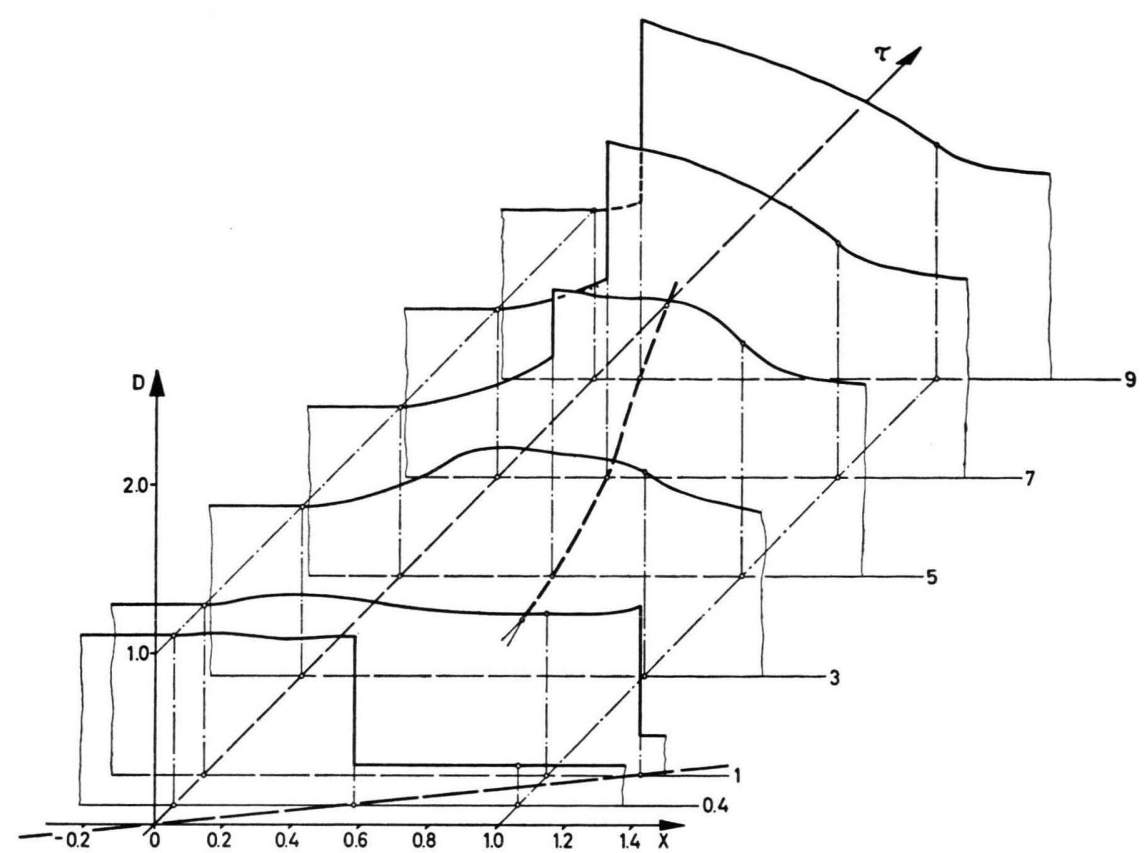

Fig. 8 b. Calculated values of dimensionless density vs. dimensionless distance and time.

The profiles show, after the jump across the shock front, first a decrease to a minimum, then an increase to higher values with increasing time at all $x$-values. In downstream direction there is, at earlier times, a rise to a maximum followed by a decrease; at later times, a decrease to a minimum followed by an increase. There is no evidence of a discontinuity indicating a secondary reflected shock front.

The velocity of the primary shock could be evaluated from the interferograms. The velocity of the shock is still not changed in the interaction region at $p_{0}=5$ Torr as compared with the case without current flowing.

\section{Results of Theoretical Investigations}

The theoretical investigations are done for the same pressure range as the experiments. For comparison the results of the 5 Torr calculations are presented here. From experimental results ${ }^{1,2}$ and the calculations ${ }^{4}$ the values

$$
\gamma, M_{1}, N_{1}, J_{\max }, K
$$

can be determined $\left(M_{1}\right.$ being used in the RankineHugoniot equations). These values for $p_{0}=5$ Torr (at $M_{\mathrm{s}}=$ shock Mach number $=10.2$ ) are as follows:

$$
\begin{array}{llll}
\gamma & =1.4 & & \text { where } \\
M_{1}=1.56 & & L \quad=7 \mathrm{~cm} \\
N_{1}=0.9 & & B_{0} \quad=6.8 \mathrm{kG} \\
J_{\max }=0.23 & & j_{\max }=120 \mathrm{~A} / \mathrm{cm}^{2} \\
K & =0.35 & & \text { are used. }
\end{array}
$$

Figure $8 \mathrm{~b}$ shows results of the calculation of the profiles of dimensionless density vs. dimensionless distance and dimensionless time. The velocity of the primary shock is practically unchanged in the interaction region. A secondary reflected shock wave forms at $X=0.48$ and $\tau=4.15$ and then propagates upstream, finally leaving the interaction region. At later $\tau$ values the dimensionless density should approach the steady-state condition, $\mathrm{cf} .{ }^{2,3}$. Behind the reflected shock the dimensionless density decreases to a minimum with increasing distance. Behind the primary shock wave the density first decreases and then increases with increasing time (for all $X$-values in the interaction region) .

\section{Discussion}

There is agreement between experimental results and theoretical predictions (Fig. 8) but only at early times after the passage of the primary shock front. The fact that the primary shock velocity is not changed also agrees with theory within the errors of 
measurement. A reflected shock, however, was not found.

At later (intermediate) times the density throughout the whole interaction region decreases to a value much lower than that behind the unperturbed primary shock. This strong expansion is in contradiction to the theory.

At still later times an increase in the density is observed with a relative minimum near to the center of the interaction region, this minimum even growing to values above that observed behind the unperturbed primary shock. This compression region certainly extends beyond both boundaries of the assumed interaction region. The increase in the upstream direction could be made consistent with the theory by assuming a reflected shock wave (which already must be outside the interaction region). The density increase in the downstream direction on the other hand is not in accordance with the theory.

A complete understanding of these phenomena is not possible at present. The current density was calculated by postulating an almost homogeneous current distribution in the asumed interaction volume. In reality the current density can be rather inhomogeneous. Therefore it is not surprising that the theoretical predictions agree with experimental results only to a limited extent. Moreover ionization effects have not been taken into acount by the theory but they might be large enough to give significant improvement in the agreement between theory and experiment.

In previous investigations ${ }^{1}$, a luminous front standing in the interaction region was observed by means of streak photographs. The results described here were produced with another measuring chamber having tungsten electrodes. With these electro- des the arcs struck immediately behind the shock front, while in the previous case they struck later in the relaxation region. Because of this the interaction force here was higher, which may sustain the assumption that a reflected shock front exists in the upstream region.

\section{Concluding Remarks}

This first comparison between calculations and measurements will be continued. The theory will be improved by taking ionization effects, and possibly radiation losses as well, into account. Furthermore, measurements of the current density should improve the physical model. Measurements of flow parameters at several different values of the interaction strength are also necessary to compare the improved theory with experimental results. But precise density measurements are only possible at high initial pressures at which the interaction strength can be raised only by raising the magnetic fields, $\mathrm{cf}^{2}$. These higher magnetic fields can be produced by field coils, as described in ${ }^{2}$, which are powered by a pulse current of $5 \mathrm{kA}$ for 1 sec. Unfortunately the available Mach-Zehner interferometer can not be used in conjunction with these coils, because it is made of ferromagnetic material. A Mach-Zehnder interferometer made of non-magnetic material is under construction and will enable us to extend our investigatios to higher magnetic fields.

The authors wish to thank Prof. Wienecke for encouragement and for his continued interest in this work and Dr. Muntenbruch for useful discussions. They are indebted to Mr. Schmid, Steffes and Loebel for valuable technical assistance. - This work was performed under the terms of the agreement on association between the Institut für Plasmaphysik and EURATOM. 